\title{
Circulating adiponectin levels are associated with peak oxygen uptake in Japanese
}

\author{
Nobuyuki Miyatake $\cdot$ Takeyuki Numata $\cdot$ Haruka Murakami $\cdot$ \\ Ryoko Kawakami $\cdot$ Kiyoshi Sanada $\cdot$ Izumi Tabata $\cdot$ \\ Motohiko Miyachi · The NEXIS Study Group
}

Received: 26 January 2014/ Accepted: 23 March 2014/Published online: 6 April 2014

(c) The Japanese Society for Hygiene 2014

\begin{abstract}
Objective The aim of this study was to investigate the link between circulating adiponectin levels and peak oxygen uptake and/or physical activity in Japanese.

Methods A total of 528 subjects (188 men and 340 women) were enrolled in this cross-sectional study. Circulating adiponectin levels, physical activity measured by tri-axial accelerometers, peak oxygen uptake and metabolic risk parameters were evaluated. We also assessed anthropometric factors, blood pressure, blood examinations and energy intake.

Results Circulating adiponectin levels were $6.7 \pm 3.0$ $\mu \mathrm{g} / \mathrm{mL}$ in men and $11.0 \pm 4.9 \mu \mathrm{g} / \mathrm{mL}$ in women. Circulating adiponectin levels were positively correlated with physical fitness after adjusting for age, physical activity evaluated by $\Sigma$ [metabolic equivalents $\times \mathrm{h}$ per week $($ METs $\mathrm{h} / \mathrm{w})$ ], cigarette smoking habit and energy intake in both sexes. However, these associations were attenuated further after adjusting for body mass index including other confounding factors, especially in men. However,
\end{abstract}

\footnotetext{
N. Miyatake ( ()

Department of Hygiene, Faculty of Medicine, Kagawa

University, Miki, Kagawa 761-0793, Japan

e-mail: miyarin@med.kagawa-u.ac.jp

T. Numata

Okayama Southern Institute of Health, Okayama Health

Foundation, Okayama 700-0952, Japan

H. Murakami - R. Kawakami - M. Miyachi

Department of Health Promotion and Exercise, National Institute of Health and Nutrition, Tokyo 162-8636, Japan

K. Sanada $\cdot$ I. Tabata

College of Sport and Health Science, Ritsumeikan University,

Kusatsu, Shiga 525-8577, Japan
}

circulating adiponectin levels were not associated with physical activity in either sex.

Conclusion Circulating adiponectin levels were associated with peak oxygen uptake rather than physical activity.

Keywords Adiponectin - Japanese - Physical activity · Peak oxygen uptake $\cdot$ Body composition

\section{Introduction}

Adiponectin, which is one of the major adipocytokines, is a 244-amino acid peptide [1]. Lower circulating adiponectin levels compared to normal controls have been observed in human subjects with obesity, type 2 diabetes mellitus and/ or cardiovascular diseases in some reports [2-4]. In the absence of pharmacological agents, increases in circulating adiponectin levels are associated with a decrease in body weight [5, 6], a reduction in lower body fat [7] and improved insulin sensitivity [8].

It is well known that physical activity and/or physical fitness induce beneficial effects on metabolic disorders [911]. Sawada et al. [12] reported that low cardiorespiratory fitness was closely linked to higher cancer mortality in Japanese men. Sandvik et al. [13] also showed that physical fitness was a graded, independent, long-term predictor of mortality from cardiovascular causes in healthy, middleaged men. Taken together, the evidence implies that physical activity and/or physical fitness tend to modify circulating adiponectin levels.

Several studies in the literature regarding the relationships between circulating adiponectin levels and physical activity and/or physical fitness have been reported [14-22]. However, the link between circulating adiponectin levels and physical activity and/or peak oxygen uptake 
independent of body composition has not fully discussed in Japanese. Therefore, in this cross-sectional study, we evaluated the relationship between circulating adiponectin levels and physical activity and/or peak oxygen uptake, which is one component of physical fitness, in apparently healthy Japanese, who are thought to have lower insulin resistance.

\section{Methods}

\section{Subjects}

We used data for 528 subjects (188 men and 340 women) among 1,118 subjects who met the following criteria: (1) wanted to volunteer in the Nutrition and Exercise Intervention Study (NEXIS) at the National Institute of Health and Nutrition, Tokyo, Japan and Okayama Southern Institute of Health, Okayama Health Foundation, Okayama, Japan; (2) had received anthropometric, physical activity, peak oxygen uptake, blood pressure (BP) measurements and blood examinations including circulating adiponectin levels; (3) received no medications for conditions such as diabetes, hypertension and dyslipidemia; and (4) provided written informed consent (Table 1).

Ethical approval for the study was obtained from the Ethical Committee of Okayama Health Foundation, Okayama, Japan and National Institute of Health and Nutrition, Tokyo, Japan. This original study protocol was registered in the ClinicalTrials.gov Identifier (NCT00926744).
Blood sampling and assays

After the subjects fasted and rested overnight for $10-12 \mathrm{~h}$, blood samples were collected to determine circulating levels of adiponectin, high-density lipoprotein (HDL) cholesterol, triglycerides (L Type Wako Triglyceride H, Wako Chemical, Osaka, Japan) and blood glucose. Circulating adiponectin levels were measured using a commercially available enzyme-linked immunosorbent assay (ELISA) kit (Otsuka Pharmaceuticals; Tokyo, Japan). Blood glucose was measured by the glucose-oxidant method.

Anthropometric and body composition measurements

Anthropometric parameters were evaluated based on the following parameters: height and body weight. Body mass index (BMI) was calculated by weight/[height $]^{2}\left(\mathrm{~kg} / \mathrm{m}^{2}\right)$.

Physical activity

The Actimarker is a small, light $(36.0 \mathrm{~g})$ and useful accelerometer. It can continuously monitor physical activity over 1 month more. Actimarker collects tri-axial acceleration data at $20 \mathrm{~Hz}$. Furthermore, the standard deviation of the data over $1 \mathrm{~min}$ is defined as the average value of acceleration. Actimarker can monitor the METs value. The METs value is calculated using a linear regression formula according to the relationship between the average value of acceleration and the METs value

Table 1 Clinical characteristics of the enrolled subjects

\begin{tabular}{|c|c|c|c|c|c|c|}
\hline & \multicolumn{3}{|l|}{ Men } & \multicolumn{3}{|l|}{ Women } \\
\hline & Mean \pm SD & Minimum & Maximum & Mean \pm SD & Minimum & Maximum \\
\hline Number of subjects & 188 & & & 340 & & \\
\hline Age (years) & $44.5 \pm 9.8$ & 30 & 64 & $48.7 \pm 9.1$ & 30 & 64 \\
\hline Height (cm) & $170.5 \pm 5.8$ & 154.3 & 192.5 & $157.6 \pm 5.5$ & 143.3 & 174.5 \\
\hline Body weight (kg) & $68.7 \pm 11.2$ & 49.9 & 168.3 & $55.0 \pm 7.9$ & 34.6 & 83.7 \\
\hline Body mass index $\left(\mathrm{kg} / \mathrm{m}^{2}\right)$ & $23.5 \pm 3.3$ & 18.2 & 54.1 & $22.1 \pm 3.1$ & 13.2 & 34.0 \\
\hline Peak oxygen uptake $(\mathrm{mL} / \mathrm{kg} / \mathrm{min})$ & $35.7 \pm 7.6$ & 12.2 & 55.8 & $29.4 \pm 6.0$ & 14.0 & 51.2 \\
\hline Physical activity (METs h/w) & $26.4 \pm 15.7$ & 1.2 & 79.8 & $27.0 \pm 15.3$ & 3.5 & 163.1 \\
\hline Systolic blood pressure (mmHg) & $122.0 \pm 13.3$ & 97.0 & 177.0 & $115.0 \pm 14.2$ & 86.0 & 191.0 \\
\hline Diastolic blood pressure (mmHg) & $75.9 \pm 9.6$ & 54.0 & 109.0 & $69.1 \pm 10.0$ & 45.0 & 104.0 \\
\hline \multicolumn{7}{|l|}{ Blood profile } \\
\hline Adiponectin $(\mu \mathrm{g} / \mathrm{mL})$ & $6.7 \pm 3.0$ & 1.8 & 17.1 & $11.0 \pm 4.9$ & 2.0 & 28.1 \\
\hline Triglyceride (mg/dL) & $108.6 \pm 64.0$ & 28.0 & 554.0 & $79.9 \pm 53.0$ & 25.0 & 589.0 \\
\hline HDL cholesterol (mg/dL) & $56.2 \pm 14.9$ & 30.0 & 149.0 & $68.2 \pm 14.6$ & 38.0 & 132.0 \\
\hline Blood glucose (mg/dL) & $93.1 \pm 11.6$ & 73.0 & 146.0 & $90.1 \pm 10.6$ & 59.0 & 188.0 \\
\hline Energy intake (kcal/day) & $2056.0 \pm 496.1$ & 763.7 & 3536.0 & $1673.8 \pm 475.5$ & 549.4 & 4301.9 \\
\hline Number of subjects with smoking habits (\%) & $33(17.6 \%)$ & & & $16(4.7 \%)$ & & \\
\hline
\end{tabular}

METs $h / w \Sigma$ [metabolic equivalents $\times$ h per week (METs $\mathrm{h} / \mathrm{w})$ ] 
measured by a gas metabolic system as previously described $[23,24]$. The subjects were instructed how to use the instrument and were told to wear it on a belt or a waist band at the right midline of the thigh from the moment they got up until they went to bed except while bathing and/or swimming, for over 2 weeks. The total amount of physical activity over 3 METs per week (METs $\mathrm{h} / \mathrm{w}$ ) was calculated using data from at least 2 weeks excluding days when subjects did not wear the Actimarker.

\section{Exercise testing}

As shown in previous report [25], after blood sampling, peak oxygen uptake was measured using a maximal graded exercise test with bicycle ergometers [Excalibur V2.0 (Okayama Southern Institute of Health), Lode BV, Groningen, The Netherlands; Monark Ergomedic 828E (National Institute and Health Nutrition), Varberg, Sweden]. The initial work load was 30-60 W, and the work rate was increased thereafter by $15 \mathrm{~W} / \mathrm{min}$ until the subject could not maintain the required pedaling frequency (60 rpm) [26]. During the latter stages of the test, each subject was verbally encouraged by operators to give his or her maximal effort. The expired gas was collected, and the rates of oxygen consumption $\left(\mathrm{VO}_{2}\right)$ and carbon dioxide production $\left(V \mathrm{CO}_{2}\right)$ were measured breath-by-breath using a cardiopulmonary gas exchange system [Oxycon Alpha (Okayama Southern Institute of Health), Mijnhrdt B.V., The Netherlands]. The expired air of subjects who were tested in the National Institute of Health and Nutrition institution was collected over 30-s intervals in Douglas bags. An oxygen and carbon dioxide mass spectrometer (Arco-1000; Arco System, Ogaki, Japan) was used to analyze oxygen and carbon dioxide concentrations. The volume of expired air was determined using a dry gas volume meter (DC-5; Shinagawa Seisakusho, Tokyo, Japan). The achievement of peak oxygen uptake was accepted if the following two conditions were met: the subject's maximal HR was $>95 \%$ of the age-predicted maximal HR (220-age), and the $V_{2}$ curve showed a leveling off.

Blood pressure (BP) measurements at rest

Resting systolic and diastolic BP (SBP and DBP) were measured indirectly using a mercury sphygmomanometer placed on the right arm of the seated participant after at least $15 \mathrm{~min}$ of rest as previously described [27].

\section{Cigarette smoking}

Cigarette smoking habit was evaluated in interviews performed by well-trained medical staff. The subjects were asked if they currently smoked cigarettes. When the answer was "yes", they were classified as current smokers. When the answer was "no", further questions were asked if they had ever smoked continuously. When the answer was "no", they were classified as non-smokers and if the answer was "yes", they were classified as previous smokers [27].

\section{Energy intake}

Energy intake (kcal/day) of enrolled subjects was evaluated by a comprehensive brief-type self-administered diet history questionnaire (BDHQ) that uses both food frequency methodology (consumption frequency and portion size) and diet history methodology (e.g. separate assessment of cooking methods and staple foods for each meal) to estimate dietary intake [28, 29].

Statistical analysis

All data are expressed as mean $\pm \mathrm{SD}$ values. Circulating adiponectin levels were not normally distributed data. Therefore, Spearman's rank correlation coefficients were calculated and used to test the significance of the relationship between parameters, where $p<0.05$ was considered statistically significant. Multiple logistic and stepwise regression analysis was also performed to test the relationship between circulating adiponectin levels and physical activity, and between circulating adiponectin levels and peak oxygen uptake.

\section{Results}

The measurements of parameters in enrolled subjects are summarized in Table 1. Circulating adiponectin levels were $6.7 \pm 3.0 \mu \mathrm{g} / \mathrm{mL}$ in men and $11.0 \pm 4.9 \mu \mathrm{g} / \mathrm{mL}$ in women. Physical activity evaluated by the Actimarker was $26.4 \pm 15.7 \mathrm{METs} \mathrm{h} / \mathrm{w}$ in men and 27.0 $\pm 15.3 \mathrm{METs} \mathrm{h} / \mathrm{w}$ in women. Peak oxygen uptake was $35.7 \pm 7.6 \mathrm{~mL} / \mathrm{kg} / \mathrm{min}$ in men and $29.4 \pm 6.0 \mathrm{~mL} / \mathrm{kg} / \mathrm{min}$ in women (Table 1).

Relationship between circulating adiponectin levels and clinical parameters was done by Spearman's rank correlation coefficient (Table 2). Circulating adiponectin levels were weakly and positively correlated with peak oxygen uptake and HDL cholesterol in men, and peak oxygen uptake, physical activity and HDL cholesterol in women (Table 2). Circulating adiponectin levels were weakly and negatively correlated with body weight, BMI and triglycerides in men; and body weight, BMI, triglycerides and blood glucose in women. However, no significant relationships between circulating adiponectin levels and other parameters were noted. 
Table 2 Relationship between circulating adiponectin levels and clinical parameters by Spearman's rank correlation coefficient
METs $h / w \Sigma$ [metabolic equivalents $\times \mathrm{h}$ per week (METs $\mathrm{h} / \mathrm{w}$ )]

Bold values are statistically significant $(p<0.05)$

\begin{tabular}{|c|c|c|c|c|}
\hline & \multicolumn{2}{|l|}{ Men } & \multicolumn{2}{|l|}{ Women } \\
\hline & rs & $p$ & rs & $p$ \\
\hline Age (years) & 0.005 & 0.9566 & 0.085 & 0.1205 \\
\hline Height $(\mathrm{cm})$ & -0.005 & 0.9449 & -0.019 & 0.7288 \\
\hline Body weight (kg) & -0.228 & 0.0018 & -0.311 & $<0.0001$ \\
\hline Body mass index $\left(\mathrm{kg} / \mathrm{m}^{2}\right)$ & -0.273 & 0.0002 & -0.325 & $<0.0001$ \\
\hline Peak oxygen uptake $(\mathrm{mL} / \mathrm{kg} / \mathrm{min})$ & 0.186 & 0.0108 & 0.229 & $<0.0001$ \\
\hline Physical activity (METs h/w) & 0.132 & 0.0714 & 0.113 & $\mathbf{0 . 0 3 7 5}$ \\
\hline Systolic blood pressure $(\mathrm{mmHg})$ & -0.090 & 0.2146 & 0.062 & 0.2594 \\
\hline Diastolic blood pressure $(\mathrm{mmHg})$ & -0.059 & 0.4139 & -0.035 & 0.5110 \\
\hline \multicolumn{5}{|l|}{ Blood profile } \\
\hline Triglyceride $(\mathrm{mg} / \mathrm{dL})$ & -0.391 & $<0.0001$ & -0.212 & $<0.0001$ \\
\hline HDL cholesterol (mg/dL) & 0.402 & $<0.0001$ & 0.434 & $<0.0001$ \\
\hline Blood glucose (mg/dL) & -0.078 & 0.2776 & -0.180 & 0.0008 \\
\hline Energy intake (kcal/day) & -0.060 & 0.4110 & -0.005 & 0.9280 \\
\hline
\end{tabular}

adiponectin levels $)=2.182+0.011($ age $)+0.017($ peak oxygen uptake) -0.042 (BMI), $\left.r^{2}=0.167, p<0.0001\right]$.

Table 3 shows the results from multiple logistic (under the median, over the median) according to quartiles of peak oxygen uptake. The odds ratios of circulating adiponectin levels according to quartiles of peak oxygen uptake were 2.382 in men and 2.745 in women (not adjusted). The odds ratios of circulating adiponectin levels according to quartiles of peak oxygen uptake were 2.822 (1.072-7.430) in men and 3.571 (1.691-7.542) in women; these were significant levels even after adjusting for age, cigarette smoking habit, physical activity and energy intake (Table 3). However, significant associations between circulating adiponectin levels and peak oxygen uptake were attenuated further after adjusting for BMI, especially in men.

The odds ratios of circulating adiponectin levels (under the median, over the median) according to quartiles of physical activity were 1.412 in men and 1.327 in women (not adjusted) (Table 4). The odds ratios of circulating adiponectin levels according to quartiles of physical activity were $1.000(0.416-2.401)$ in men and 0.704 (0.354-1.398) in women after adjusting for age, cigarette smoking habit, peak oxygen uptake and energy intake (Table 4). These associations between circulating adiponectin levels and physical activity in both sexes did not change further after adjusting for BMI.

Finally, we used stepwise multiple regression analysis to evaluate the effect of clinical parameters, i.e. age, BMI, energy intake, physical activity and peak oxygen uptake on circulating adiponectin levels [ $\ln$ (Circulating adiponectin levels)], and found that only BMI was significant in men $[\ln$ (Circulating adiponectin levels) $=2.445-0.027$ (BMI), $r^{2}=0.039, p=0.0069$ ], while age, peak oxygen uptake and BMI were significant in women [ $\ln ($ Circulating

\section{Discussion}

In this study, we accurately evaluated the relationship between circulating adiponectin levels and peak oxygen uptake and/or physical activity using a tri-axial accelerometer in Japanese subjects. Peak oxygen uptake rather than physical activity was closely associated with circulating adiponectin levels.

In some studies in the literature, the effect of exercise intervention on adiponectin levels has been reported. Hara et al. [15] reported that with an 8-week training program adiponectin levels were unchanged in young obese men. Kobayashi et al. [16] also showed the effect of walking with a pedometer on adiponectin levels, and noted that changes in adiponectin levels were not observed in middleaged Japanese men. However, in obese young women, exercise reduced body weight, BMI, body fat percentage and increased adiponectin with a 7-month exercise training program [17]. Tsukinoki et al. [19] also showed using a self-administered questionnaire about their daily lives that physical exercise two or more times a week was related to adiponectin levels in Japanese male workers. Very few of these previous studies accurately evaluated the relationship between physical activity and/or peak oxygen uptake and circulating adiponectin levels independent of body composition.

In this study, we accurately evaluated peak oxygen uptake and physical activity using a tri-axial accelerometer in Japanese subjects who were not taking any medication, and found that peak oxygen uptake, rather than physical 
Table 3 Odds ratios of adiponectin levels according to quartiles of peak oxygen uptake

Data were analyzed by multiple logistic regression analysis

Model 1 not adjusted

Model 2 adjusted for age

Model 3 adjusted for age, cigarette smoking habit and physical activity

Model 4 adjusted for age, cigarette smoking habit, physical activity and energy intake

Model 5 adjusted for age, cigarette smoking habit, physical activity, energy intake and BMI

Bold value indicates that the $95 \%$ confidence interval does not cross 1

Table 4 Odds ratios of adiponectin levels according to quartiles of physical activity

METs $h / w \Sigma$ [metabolic equivalents $\times \mathrm{h}$ per week (METs h/w)]

Data were analyzed by multiple logistic regression analysis

Model 1 not adjusted

Model 2 adjusted for age

Model 3 adjusted for age, cigarette smoking habit and peak oxygen uptake

Model 4 adjusted for age, cigarette smoking habit, peak oxygen uptake and energy intake

Model 5 adjusted for age, cigarette smoking habit, peak oxygen uptake, energy intake and BMI

\begin{tabular}{|c|c|c|c|c|}
\hline \multicolumn{5}{|c|}{ Quartiles of peak oxygen uptake (mL/kg/min) } \\
\hline & Q1 & Q2 & Q3 & Q4 \\
\hline \multicolumn{5}{|l|}{ Men } \\
\hline Number of subjects & 47 & 47 & 47 & 47 \\
\hline Mean + SD & $26.5 \pm 3.7$ & $32.9 \pm 1.5$ & $37.8 \pm 1.6$ & $45.4 \pm 4.2$ \\
\hline Model 1 & 1 & $1.000(0.441-2.266)$ & $1.409(0.625-3.177)$ & $2.382(1.039-5.462)$ \\
\hline Model 2 & 1 & $1.119(0.485-2.582)$ & $1.730(0.731-4.093)$ & $3.155(1.268-7.849)$ \\
\hline Model 3 & 1 & $1.127(0.483-2.628)$ & $1.516(0.613-3.753)$ & $2.748(1.047-7.213)$ \\
\hline Model 4 & 1 & $1.123(0.481-2.624)$ & $1.600(0.642-3.989)$ & $2.822(1.072-7.430)$ \\
\hline Model 5 & 1 & $0.884(0.367-2.131)$ & $1.239(0.481-3.191)$ & $2.055(0.746-5.661)$ \\
\hline \multicolumn{5}{|l|}{ Women } \\
\hline Number of subjects & 85 & 85 & 85 & 85 \\
\hline Mean \pm SD & $22.3 \pm 2.3$ & $26.9 \pm 1.0$ & $30.9 \pm 1.4$ & $37.3 \pm 3.6$ \\
\hline Model 1 & 1 & $1.343(0.725-2.485)$ & $2.488(1.342-4.616)$ & $2.745(1.475-5.107)$ \\
\hline Model 2 & 1 & $1.514(0.808-2.838)$ & $3.125(1.633-5.979)$ & $4.161(2.068-8.374)$ \\
\hline Model 3 & 1 & $1.429(0.757-2.696)$ & $2.886(1.491-5.586)$ & $3.481(1.653-7.327)$ \\
\hline Model 4 & 1 & $1.452(0.768-2.748)$ & $2.892(1.492-5.605)$ & $3.571(1.691-7.542)$ \\
\hline Model 5 & 1 & $1.104(0.564-2.163)$ & $2.100(1.048-4.209)$ & $2.068(0.932-4.590)$ \\
\hline
\end{tabular}

\begin{tabular}{|c|c|c|c|c|}
\hline \multicolumn{5}{|c|}{ Quartiles of physical activity (METs h/w) } \\
\hline & Q1 & Q2 & Q3 & Q4 \\
\hline \multicolumn{5}{|l|}{ Men } \\
\hline Number of subjects & 47 & 47 & 47 & 47 \\
\hline Mean + SD & $10.1 \pm 3.3$ & $19.5 \pm 2.6$ & $28.2 \pm 2.5$ & $47.6 \pm 13.8$ \\
\hline Model 1 & 1 & $0.543(0.238-1.240)$ & $1.089(0.485-2.447)$ & $1.412(0.624-3.195)$ \\
\hline Model 2 & 1 & $0.546(0.239-1.247)$ & $1.083(0.481-2.435)$ & $1.404(0.620-3.180)$ \\
\hline Model 3 & 1 & $0.510(0.220-1.185)$ & $0.875(0.375-2.040)$ & $1.038(0.435-2.479)$ \\
\hline Model 4 & 1 & $0.468(0.199-1.102)$ & $0.838(0.358-1.961)$ & $1.000(0.416-2.401)$ \\
\hline Model 5 & 1 & $0.435(0.181-1.044)$ & $0.830(0.350-1.971)$ & $0.991(0.407-2.408)$ \\
\hline \multicolumn{5}{|l|}{ Women } \\
\hline Number of subjects & 85 & 85 & 85 & 85 \\
\hline Mean + SD & $13.0 \pm 3.4$ & $21.4 \pm 1.7$ & $28.5 \pm 2.4$ & $44.9 \pm 19.2$ \\
\hline Model 1 & 1 & $1.099(0.601-2.010)$ & $1.605(0.876-2.940)$ & $1.327(0.726-2.426)$ \\
\hline Model 2 & 1 & $1.086(0.593-1.988)$ & $1.577(0.859-2.895)$ & $1.318(0.720-2.411)$ \\
\hline Model 3 & 1 & $0.989(0.527-1.858)$ & $1.099(0.577-2.092)$ & $0.706(0.356-1.401)$ \\
\hline Model 4 & 1 & $0.998(0.530-1.880)$ & $1.081(0.567-2.062)$ & $0.704(0.354-1.398)$ \\
\hline Model 5 & 1 & $1.320(0.674-2.584)$ & $1.147(0.591-2.224)$ & $0.817(0.404-1.650)$ \\
\hline
\end{tabular}

activity, was associated with circulating adiponectin levels. However, only in the partial population (grade Q3 of peak oxygen uptake), peak oxygen uptake was associated with circulating adiponectin levels in women after adjusting for BMI. Additionally, the significance of the relationships between circulating adiponectin levels and peak oxygen uptake disappeared in men further after adjusting for BMI. Using stepwise regression analysis, age, peak oxygen uptake and BMI were determinant factors of circulating adiponectin levels in women. In the line of evidence, peak oxygen uptake rather than physical activity was associated with circulating adiponectin levels independent of BMI and it was unique feature in women. Martinez-Gomez et al. [14] evaluated the independent and joint associations of physical activity and cardiorespiratory fitness and muscular fitness with adiponectin in adolescents. They found that physical activity was not significantly associated with adiponectin, while vigorous physical activity showed a significant inverse association with adiponectin after adjusting for age, sex, pubertal status and abdominal 
circumference [14]. High-intensity interval training positively changed adiponectin levels in obese adolescent girls [18]. In addition, circulating adiponectin levels were correlated with body weight, BMI, triglyceride and HDL cholesterol as previously reported $[30,31]$. Taken together, peak oxygen uptake rather than physical activity may be closely linked to circulating adiponectin levels. Therefore, increasing peak oxygen uptake may be useful for improving circulating adiponectin levels independent of BMI.

There were some potential limitations in this study. First, our study was a cross-sectional rather than a longitudinal study, so the effect of long-term physical activity and/or physical fitness could not be evaluated clearly. Second, 528 subjects in our study voluntarily underwent measurements: they were therefore more likely to be health conscious than the average person. Third, the Actimarker cannot evaluate isometric muscle action and only evaluates a partial aspect of physical activity. Fourth, we could not show any gender difference or a clear mechanism between circulating adiponectin levels and peak oxygen uptake. A difference in reference values of peak oxygen uptake, physical activity and circulating adiponectin levels between men and women may have induced the gender differences in this study. In fact, circulating adiponectin levels in women were higher than those in men, while peak oxygen uptake in men was higher than that in women. Genotype GT + TT at SNP276(G/T) subjects showed a greater adiponectin-related response to exercise training than those with the GG genotype [32]. In addition, high molecular weight (HMW) adiponectin is reported to be an active form of protein [33] and we could not evaluate this as done in a previous study [33]. However, it seems reasonable to suggest that promoting peak oxygen uptake will result in increasing circulating adiponectin levels independent of BMI in clinical practice. To confirm these findings, further longitudinal and larger sample size studies are required in the Japanese population.

Acknowledgments This research was supported in part by Research Grants from the Ministry of Health, Labor, and Welfare of Japan.

Conflict of interest The authors declare no conflict of interests.

\section{References}

1. Yamauchi T, Kamon J, Waki H, Terauchi Y, Kubota N, Hara K, et al. The fat-derived hormone adiponectin reverses insulin resistance associated with both lipoatrophy and obesity. Nat Med. 2001;7:941-6.

2. Dekker JM, Funahashi T, Nijpels G, Pilz S, Stehouwer CD, Snijder MB, et al. Prognostic value of adiponectin for cardiovascular disease and mortality. J Clin Endocrinol Metab. 2008;93:1489-96.

3. Jurimae J, Jurimae T, Ring-Dimitriou S, LeMura LM, Arciero PJ, von Duvillard SP. Plasma adiponectin and insulin sensitivity in overweight and normal weight middle-aged premenopausal women. Metabolism. 2009;58:638-43.

4. Matsuzawa Y, Funahashi T, Kihara S, Shimomura I. Adiponectin and metabolic syndrome. Aterioscler Thromb Vasc Biol. 2004;24:29-33.

5. Boudou P, Sobngwi E, Mauvais-Jarvis F, Vexiau P, Gautier J-F. Absence of exercise-induced variations in adiponectin levels despite decreased abdominal adiposity and improved insulin sensitivity in type 2 diabetic men. Eur $\mathrm{J}$ Endocrinol. 2003;149:421-4.

6. Esposito K, Pontillo A, Di Palo C, Giugliano G, Masella M, Marfella R, et al. Effect of weight loss and lifestyle changes on vascular inflammatory markers in obese women. JAMA. 2003;289:1799-804.

7. Buemann B, Sorensen TI, Pedersen O, Black E, Holst C, Toubro $\mathrm{S}$, et al. Lower-body fat mass as an independent marker of insulin sensitivity - the role of adiponectin. Int J Obes. 2005;29:624-31.

8. Tshritter O, Fritsche A, Thamer C, Haap M, Shirkavand F, Rahe St, et al. Plasma adiponectin concentrations predict insulin sensitivity of both glucose and lipid metabolism. Diabetes. 2003;52:239-43.

9. Miyatake N, Takahashi K, Wada J, Nishikawa H, Morishita A, Suzuki H, et al. Daily exercise lowers blood pressure and reduces visceral fat in overweight Japanese men. Diabetes Res Clin Pract. 2003;62:149-57.

10. Yamanouchi K, Shinozaki T, Chikada K, Nishikawa T, Ito K, Shimizu $S$, et al. Daily walking combined with diet therapy is a useful means for obese NIDDM patients not only to reduce body weight but also to improve insulin sensitivity. Diabetes Care. 1995;18:775-8.

11. Miyatake N, Nishikawa H, Morishita A, Kunitomi M, Wada J, Suzuki $\mathrm{H}$, et al. Daily walking reduces visceral adipose tissue areas and improves insulin resistance in Japanese obese subjects. Diabetes Res Clin Pract. 2002;58:101-7.

12. Sawada SS, Muto T, Tanaka H, Lee IM, Paffenbager RS Jr, Shindo M, et al. Cardiorespiratory fitness and cancer mortality in Japanese men: a prospective study. Med Sci Sports Exerc. 2003;35:1546-50.

13. Sandvik L, Erikssen J, Thaulow E, Erikssen G, Mundal R, Rodahl K. Physical fitness as a predictor of mortality among healthy, middle-aged Norwegian men. N Engl J Med. 1993;328:533-7.

14. Martinez-Gomez D, Eisenmann JC, Gomez-Martinez S, Veses A, Romeo J, Veiga OL, AFINOS Study Group, et al. Associations physical activity and fitness with adipocytokines in adolescents: the AFINOS Study. Nutr Metab Cardiovasc Dis. 2012;22:252-9.

15. Hara T, Fujiwara H, Nakao H, Mimura T, Yoshikawa T, Fujimoto $\mathrm{S}$. Body composition is related to increase in plasma adiponectin levels rather than training in young obese men. Eur $\mathbf{J}$ Appl Physiol. 2005;94:520-6.

16. Kobayashi J, Murase Y, Asano A, Nohara A, Kawashiri MA, Inazu A, et al. Effect of walking with a pedometer on serum lipid and adiponectin levels in Japanese middle-aged men. J Atheroscler Thromb. 2006;13:197-201.

17. Kondo T, Kobayashi I, Murakami M. Effect of exercise on circulating adipokine levels in obese young women. Endocr J. 2006;53:189-95.

18. Racil G, Ben Ounis O, Hammouda O, Kallel A, Zouhal H, Chamari K, et al. Effects of high vs. moderate exercise intensity during interval training on lipids and adiponectin levels in obese young females. Eur J Appl Physiol. 2013;113:2531-40.

19. Tsukinoki R, Morimoto K, Nakayama K. Association between lifestyle factors and plasma adiponectin levels in Japanese men. Lipids Health Dis. 2005;4:27.

20. Jurimae J, Kums T, Jurimae T. Plasma adiponectin concentration is associated with the average accelerometer daily steps counts in healthy elderly females. Eur J Appl Physiol. 2010;109:823-8. 
21. Ring-Dimitriou S, Paulweber B, von Duvillard SP, Stadlmann M, LeMura LM, Lang J, Muller E. The effect of physical activity and physical fitness on plasma adiponectin in adults with predisposition to metabolic syndrome. Eur J Appl Physiol. 2006;98: 472-81.

22. Ferguson MA, White LJ, McCoy S, Kim HW, Petty T, Wilsey J. Plasma adiponectin response to exercise in healthy subjects. Eur J Appl Physiol. 2004;91:324-9.

23. Matsumura Y, Yamamoto M, Kitado T, Nakamura H, Kidera K, Fujimoto S. High-accuracy physical activity monitor utilizing three-axis accelerometer. Natl Tech Rep. 2008;56:60-6 (in Japanese).

24. Sugio A, Minakata Y, Kanda M, Akamatsu K, Koarai A, Hirano $\mathrm{T}$, et al. Validation of a compact motion sensor for the measurement of physical activity in patients with chronic obstructive pulmonary disease. Respiration. 2012;83:300-7.

25. Cao ZB, Miyatake N, Higuchi M, Miyachi M, Ishikawa-Takata $\mathrm{K}$, Tabata I. Predicting $\mathrm{VO}_{2 \max }$ with and objectively measured physical activity in Japanese women. Med Sci Sports Exerc. 2010;42:179-86.

26. Miyachi M, Tanaka H, Yamamoto K, Yoshioka A, Takahashi K, Onodera S. Effects of one-legged endurance training on femoral arterial and venous size in healthy humans. J Appl Physiol. 2001;90:2439-44.

27. Miyatake N, Wada J, Nakatsuka A, Sakano N, Teshigawara S, Miyachi M, et al. Serum vaspin levels are associated with physical activity or physical fitness in Japanese: a pilot study. Environ Health Prev Med. 2014. doi:10.1007/s12199-013-0375-1.
28. Sasaki S, Yanagibori R, Amano K. Self-administrated diet history questionnaire developed for health education: a relative validation of the test-version by comparison with 3-day diet record in women. J Epidemiol. 1998;8:203-15.

29. Kobayashi S, Murakami K, Sasaki S, Okubo H, Hirota N, Notsu A, et al. Comparison of relative validity of food group intakes estimated by comprehensive and brief-type self-administered diet history questionnaires against $16 \mathrm{~d}$ dietary records in Japanese adults. Public Health Nutr. 2011;14:1200-11.

30. Okauchi Y, Kishida K, Funahashi T, Noguchi M, Ogawa T, Ryo $\mathrm{M}$, et al. Changes in serum adiponectin concentrations correlate with changes in BMI, waist circumference, and estimated visceral fat area in middle-aged general population. Diabetes Care. 2009;32:e122.

31. Komatsu M, Ohfusa H, Aizawa T, Hashizume K, Hashizume K. Adiponectin inversely correlates with high sensitive C-reactive protein and triglycerides, but not with insulin sensitivity, in apparently healthy Japanese women. Endocr J. 2007;54:553-8.

32. Huang H, Tada lida K, Murakami H, Saito Y, Otsuki T, Iemitsu $\mathrm{M}$, et al. Influence of adiponectin gene polymorphism SNP276 $(\mathrm{G} / \mathrm{T})$ on adiponectin in response to exercise training. Endocr J. 2007;54:879-86.

33. Ando D, Hosaka Y, Suzuki K, Yamagata Z. Effects of exercise training on circulating high molecular weight adiponectin and adiponectin oligomer composition: a randomized controlled trial. J Atheroscler Thromb. 2009;16:733-9. 